\title{
POSITIVE PERIODIC SOLUTIONS FOR THE NONLINEAR WAVE EQUATION
}

\section{SvetLin Georgiev Georgiev}

Abstract. In this paper we prove that nonlinear wave equation

$$
u_{t t}-\Delta u=f\left(t, x, u, u_{t}, u_{x}\right)
$$

has unique positive solution $u(t, x)$ which is $\omega$-periodic with respect to the time variable $t$. The period $\omega>0$ is arbitrarily chosen and fixed.

Mathematics subject classification (2010): 35L05, 35L20, 35L40.

Keywords and phrases: Nonlinear wave equation, periodic solutions.

\section{REFERENCES}

[1] B. Biondi, G. Palacharla, 3-D-prestack migration of common-azimuth data, Geophysics, 61, 6 (1996), 1822-1842.

[2] H. BREZIS, Periodic solutions of nonlinear vibrating strings and duality principles, Bull. Amer. Math. Soc. (NS), 8, 3 (1983), 409-426.

[3] S. Georgiev, Positive periodic solutions for the Korteweg de Vries equation, Electron. J. Differ. Equ., 2007, 49 (2007), 13p.

[4] S. Geor GIEv, Positive periodic solutions for the nonlinear parabolic equation, Far East J. Dyn. Syst., 9, 3 (2007), 455-512.

[5] S. GeORGIEv, On the integrating seismic facies and petro-acoustic modeling, (in preparation)

[6] M. KRASNOSEL'SKII, P. ZABREIKo, Geometrical methods of nonlinear analysis, A Series of Comprehensive studies in Mathematics 263, Springer-Verlag, Berlin-Heidelberg-New York-Tokio, 1984.

[7] M. K. Kwong, On Karsnoselskii's cone fixed point theorem, Fixed Point Theory Appl., 2008, Article ID 164537, (2008), 18p.

[8] R. PYKE, M. SIGAL, Nonlinear wave equations: constraints on periods and exponential bounds for periodic solutions, Duke Mathematical Journal, 88 (1997), 133-180.

[9] P. Rabinowitz, Periodic solutions of nonlinear partial differential equations, Comm. Pure Appl. Math., 20 (1967), 145-205.

[10] R. Stolt, A. Benson, Seismic migration-theory and practice, Geophysical Press, LondonAmsterdam, 1986.

[11] O. VeJVodA, Periodic solutions of linear and weakly nonlinear wave equation in one dimension, I, Czech. Math. J., 14 (1964), 341-382.

[12] J. Xiaofeng, H. Tianyue, W. RunQiu, A meshless method for acoustic and elastic modeling, Appl. Geophysics, 2, 1 (2005). 